\title{
An elevated IgG4 response in chronic infectious aortitis is associated with aortic atherosclerosis
}

\author{
Zakir Siddiquee ${ }^{1}$, R Neal Smith ${ }^{1}$ and James R Stone ${ }^{1,2}$ \\ ${ }^{1}$ Department of Pathology, Massachusetts General Hospital, Harvard Medical School, Boston, MA, USA and \\ ${ }^{2}$ Center for Systems Biology, Massachusetts General Hospital, Boston, MA, USA
}

\begin{abstract}
Recently, it was shown that infectious bacterial aortitis can stimulate an elevated lgG4 ${ }^{+}$plasma cell response in the vessel wall, which could mimic IgG4 aortitis/periaortitis. However, the factors that are associated with an elevated IgG4 ${ }^{+}$plasma cell response in infectious aortitis are unclear. To ascertain these factors, 17 cases of infectious aortitis and 6 cases of non-infectious severe abdominal aortic atherosclerosis were assessed for the magnitude of $\mathrm{IgG4}^{+}$plasma cell response. The degree of $\operatorname{lgG} 4^{+}$plasma cell infiltration was determined by immunohistochemistry. Infectious cases were subcharacterized as chronic ( $>3$ weeks duration) or acute ( $<3$ weeks duration) based on the duration of symptoms, and as involving either the ascending aorta or the distal aorta, ie, the descending thoracic and/or abdominal aorta. There was a 5-16-fold greater degree of IgG4+ plasma cell infiltration in the chronic distal infectious aortitis group compared with the other three infectious aortitis groups $(P \leq \mathbf{0 . 0 0 0 7 )}$, and compared with non-infectious severe abdominal aortic atherosclerosis $(P<0.0008)$. This resulted in a greater IgG4/lgG ratio in the chronic distal infectious aortitis group compared with the acute ascending and acute distal infectious aortitis groups $(P<0.03)$. The degree of IgG4 ${ }^{+}$plasma cell infiltration in chronic distal infectious aortitis overlaps with that seen in the aortitis and periaortitis of IgG4-related disease. In the chronic infectious aortitis cases, the degree of IgG4+ plasma cell infiltration was more intense in patients with moderate to severe aortic atherosclerosis compared with those patients with less aortic atherosclerosis $(P=0.007)$. These findings indicate that an elevated lgG4+ ${ }^{+}$plasma cell response occurs in the descending thoracic and abdominal aorta in the setting of chronic bacterial infectious aortitis and pre-existing atherosclerosis. This inflammatory response to chronic infection in atherosclerosis-laden aortas may have implications for the development of IgG4-rich inflammatory atherosclerotic aortic aneurysms.

Modern Pathology (2015) 28, 1428-1434; doi:10.1038/modpathol.2015.105; published online 4 September 2015
\end{abstract}

IgG4-related aortitis/periaortitis is a form of noninfectious aortitis that is distinguished from giant cell aortitis and Takayasu aortitis by a lymphoplasmacytic pattern of inflammation rather than a granulomatous/ giant cell pattern of inflammation, and by increased numbers of IgG4-expressing plasma cells. ${ }^{1-6}$ IgG4-related aortitis/periaortitis appears to occur in two forms. ${ }^{6}$ In some cases, there is an outright aortitis with evidence of systemic IgG4-related disease including elevated serum levels of IgG4. Such cases occur with or without the presence of concurrent aortic atherosclerosis. In other cases, the disease appears to be isolated to a defined aortic segment

Correspondence: Dr JR Stone, MD, PhD, Department of Pathology, Massachusetts General Hospital, Harvard Medical School, Simches Research Building Room 8236, 185 Cambridge Street CPZN, Boston, MA 02114, USA.

E-mail: jrstone@partners.org

Received 10 June 2015; revised 22 July 2015; accepted 23 July 2015; published online 4 September 2015 without evidence of systemic involvement of elevation of serum IgG4 levels. These latter cases typically involve aneurysmal segments of the abdominal aorta that contain severe atherosclerosis and have a histologic appearance that in fact overlaps with that of severe atherosclerosis. Such cases are also referred to as IgG4-rich inflammatory atherosclerotic aortic aneurysms. Since the presence of an elevated IgG4 ${ }^{+}$ plasma cell infiltrate is a critical feature for the pathologic diagnosis of this entity, it is important to understand the factors that can stimulate such an inflammatory response in the aortic wall.

Recently, some cases of infectious bacterial aortitis were shown to have elevated levels of $\mathrm{IgG}^{+}$plasma cells. ${ }^{7}$ In these cases, the degree of IgG4 ${ }^{+}$plasma cell infiltration were such that the cases would satisfy international IgG4 staining criteria for IgG4-related aortitis. Patients with IgG4-related disease are frequently treated with immunosuppression. ${ }^{8}$ Thus, it is important to be able to distinguish IgG4-related 
aortitis from infectious forms of aortitis, and to understand the factors that drive an elevated IgG4 response in the setting of infectious aortitis. To address this issue, we have analyzed the immunohistochemical staining of IgG and IgG4 in 17 cases of infectious aortitis, the largest series of infectious aortitis analyzed for IgG4 staining that has been reported to date.

\section{Materials and methods}

\section{Case Selection}

Cases were selected from both autopsies and surgical specimens obtained during the 9-year period from January 2006 to January 2015. Inclusion criteria were the presence of active suppurative infectious aortitis with bacteria identified on Gram, Grocott's methenamine silver, or Steiner stain and the availability of tissue for analysis. ${ }^{1,9}$ Patients with prior aortic surgery or endovascular graft placement at the site of infection were excluded. Seventeen cases met the study criteria. Three of these cases have been reported previously. ${ }^{7,10}$ The results of microbial culture of either peripheral blood or explanted aortic tissue and antibiotic therapy were obtained from the clinical records. Six consecutive patients, without infectious aortitis, who underwent resection of the abdominal aorta for complications of atherosclerosis (aneurysm $n=3$, luminal thrombosis $n=3$ ) between January 2010 and May 2013 were included as a separate control group in a secondary analysis. The study was approved by the institution's human subject's institutional review board.

\section{Immunohistochemistry and Atherosclerosis Grading}

Immunohistochemical staining for IgG and IgG4 was performed on formalin-fixed paraffin-embedded tissue, as described previously.,11 The number of cells expressing IgG and IgG4 were counted using a $\times 400$ high-power field (hpf) measuring $0.16 \mathrm{~mm}^{2}$. Immunohistochemical staining for IgG4 and IgG was quantified following international consensus guidelines. ${ }^{12}$ For each case, the cells in three nonoverlapping $\times 400 \mathrm{hpfs}$ were counted, and the results averaged. The hpfs showing the highest number of IgG4-expressing plasma cells were selected for counting. The degree of aortic atherosclerosis was scored as described previously: Grade 0: intimal hyperplasia and fatty streaks; Grade 1: intermediate lesions and atheromas without fibrosis (AHA Grade III and IV lesions); Grade 2: fibroatheromas with scarring involving $<1 / 3$ of the media; Grade 3 : fibroatheromas with scarring involving $\geq 1 / 3$ of the media. ${ }^{1}$

\section{Data Analysis and Statistical Methods}

The cases were divided into four patient groups based on location and duration of symptoms. For location, the patients were classified as having

Table 1 Infectious aortitis patient groups based on duration of symptoms and aortic segment involved

\begin{tabular}{|c|c|c|c|c|}
\hline & \multicolumn{2}{|c|}{ Ascending } & \multicolumn{2}{|c|}{ Distal $^{\mathrm{a}}$} \\
\hline & Acute & Chronic & Acute & Chronic \\
\hline $\mathrm{N}$ & 4 & 4 & 4 & 5 \\
\hline Age, vrs (mean \pm s.d.) & $70 \pm 10$ & $57 \pm 20$ & $72 \pm 6$ & $70 \pm 10$ \\
\hline Gender $(\mathrm{M} / \mathrm{F})$ & $3 / 1$ & $2 / 2$ & $1 / 3$ & $4 / 1$ \\
\hline Duration, days (mean \pm s.d.) & $10 \pm 4$ & $68 \pm 40$ & $9 \pm 4$ & $60 \pm 22$ \\
\hline \multirow[t]{3}{*}{ Organisms } & S. aureus $\times 2$ & S. aureus $\times 3$ & S. aureus $\times 2$ & S. aureus \\
\hline & Streptococcus mitis & $\mathrm{GPC}^{\mathrm{b}}$ & $\begin{array}{c}\text { GPC } \\
\text { mixed }^{\mathrm{C}}\end{array}$ & Group B Streptococcus \\
\hline & & & & $\begin{array}{c}\text { Pasteurella multocida } \\
\text { GPC }\end{array}$ \\
\hline \multirow{12}{*}{$\begin{array}{l}\text { Antibiotics received } \\
\text { before surgery or death }\end{array}$} & Cefepime & Atovaquone & Cefazolin & Cefazolin \\
\hline & ceftriaxone $\times 4$ & daptomycin & cefepime $\times 3$ & cefepime $\times 3$ \\
\hline & clindamycin & gentamicin & ciprofloxacin & ceftriaxone $\times 3$ \\
\hline & doxycycline & levofloxacin $\times 2$ & imipenem $\times 2$ & clindamycin \\
\hline & $\begin{array}{l}\text { gentamicin } \times 2 \\
\text { nafcillin }\end{array}$ & $\begin{array}{c}\text { linezolid } \\
\text { metronidazole }\end{array}$ & $\begin{array}{c}\text { levofloxacin } \times 2 \\
\text { linezolid }\end{array}$ & $\begin{array}{l}\text { daptomycin } \\
\text { imipenem }\end{array}$ \\
\hline & penicillin & nafcillin $\times 2$ & metronidazole & levofloxacin \\
\hline & vancomycin $\times 4$ & telvancin & piperacillin $\times 2$ & linezolid \\
\hline & & vancomycin $\times 3$ & tazobactam × 2 & metronidazole $\times 2$ \\
\hline & & & vancomycin $\times 3$ & piperacillin × 2 \\
\hline & & & & $\begin{array}{l}\text { sulmethoxazole } \\
\text { tazobactam } \times 2\end{array}$ \\
\hline & & & & telvancin \\
\hline & & & & trimethoprim \\
\hline & & & & $\cos y=\operatorname{cin} x$ \\
\hline
\end{tabular}

\footnotetext{
${ }^{\mathrm{a} D i s t a l}$ : descending thoracic and/or abdominal aorta. ${ }^{\mathrm{b}} \mathrm{GPC}$ : Gram positive cocci not further characterized. ${ }^{\mathrm{c}}$ Mixed: Enterococcus and Lactobacillus.
} 
involvement of either the ascending aorta or the distal aorta, the latter of which encompasses the descending thoracic aorta and/or abdominal aorta. For duration, the time interval between the onset of symptoms and the surgery or autopsy was determined. A duration of $<3$ weeks was defined as acute, and a duration of $>3$ weeks was defined as chronic. Immunohistochemical staining was compared across the patient groups using ANOVA with post-test by Bonferroni. Atherosclerosis scores were compared using the Mann-Whitney $U$-test or $t$-test, as indicated. In secondary analyses, potential associations of IgG4 staining with age were assessed by Spearman rank-order correlation, and potential associations with gender and type of bacteria were assessed by $t$-test. $P$-values $<0.05$ were considered significant.

\section{Results}

\section{Immunohistochemical Staining}

The patient characteristics for the 17 patients in the four infectious aortitis groups are shown in Table 1, along with the specific bacteria identified and antibiotics received. There was a marked difference in the degree of IgG4 staining between the four patient groups. The numbers of adventitial $\operatorname{IgG}^{+}$ plasma cells were relatively low in both of the acute infectious aortitis groups and in the chronic ascending infectious aortitis group (Figures 1 and 2). However, there was an 8-16-fold increase in the number of adventitial $\operatorname{IgG}^{+}$plasma cells in the chronic distal infectious aortitis group compared with the other three infectious aortitis groups $(P \leq 0.0007)$, with this group having a mean of 80 IgG4 ${ }^{+}$plasma cells per hpf. There was a similar, but less intense, increase in the number of adventitial $\mathrm{IgG}^{+}$plasma cells in the chronic distal infectious aortitis group compared with the two acute infectious aortitis groups. The $\mathrm{IgG} 4 / \mathrm{IgG}$ ratio was also increased in the chronic distal infectious aortitis group compared with the two ascending aortitis groups, with the chronic distal infectious aortitis group having a mean $\operatorname{IgG} 4 / \operatorname{IgG}$ ratio of $46 \%$.

The international criteria for diagnosing IgG4related disease in the aorta requires an $\operatorname{IgG} 4 / \operatorname{IgG}$ ratio $>50 \%$ and more than $50 \mathrm{IgG}^{+}$plasma cells/ hpf. $^{12}$ Two of the five cases of chronic distal infectious aortitis would satisfy staining criteria for IgG4-related disease. If the more generally applied criteria using an IgG4/IgG ratio of $>40 \%$ were employed, then three of the five cases would satisfy staining criteria for IgG4-related disease. All five cases had obstructed veins in the inflamed adventitia along with scarring, but none of the cases displayed true storiform fibrosis with a pinwheel pattern. Serum IgG4 levels were not available for any of the five patients with chronic infectious aortitis. None of the cases in the other three infectious aortitis groups would satisfy either staining criteria for IgG4-related disease.

In contrast to the marked association of $\operatorname{IgG} 4^{+}$ plasma cell infiltration with location and chronicity, the IgG4 ${ }^{+}$plasma cell counts in this cohort were not associated with age or gender for all 17 cases, for the 9 chronic infectious aortitis cases, or for the 9 distal infectious aortitis cases. Likewise, there was no association of the $\mathrm{IgG}^{+}$plasma cell counts with Staphylococcus aureus infection compared with the other identified infectious agents.

\section{Association with Atherosclerosis}

To ascertain possible reasons for the specific increase in $\operatorname{IgG}^{+}$plasma cells in the chronic distal infectious aortitis group compared with the chronic ascending infectious aortitis group, the aortic morphology in the distal aorta groups was compared with that in the ascending aorta groups. There was significantly more severe aortic atherosclerosis in the distal infectious aortitis cases compared with the proximal infectious aortitis cases (Figure 3). Furthermore, for all nine patients with chronic infectious aortitis, the degree of $\operatorname{IgG}^{+}$plasma cell infiltration was significantly more intense for the patients with Grade 2 or 3 aortic atherosclerosis compared with the patients with Grade 0 or 1 aortic atherosclerosis (71 \pm 37 vs $5 \pm 3$ cells/hpf, $P=0.007$, $t$-test). Thus, the increased $\mathrm{IgG}^{+}$plasma cell infiltration in chronic infectious aortitis occurs in the setting of more advanced atherosclerosis.

The acute distal infectious aortitis group had an overall atherosclerosis score of $2.75 \pm 0.25$ (mean \pm s.e.), but did not show the high $\operatorname{IgG}^{+}$plasma cell infiltration seen in the chronic distal infectious aortitis group, suggesting that the elevated $\operatorname{IgG} 4^{+}$ plasma cell infiltration in these cases requires both the presence of atherosclerosis and chronic infection. To verify this observation, the aortas from six patients who underwent resection of the abdominal aorta for complications of atherosclerosis in the absence of infectious aortitis were also evaluated for IgG4 and IgG staining. The group consisted of three men and three women with an age of $65 \pm 10$ years (mean \pm s.d.), and all six cases displayed Grade 3 atherosclerosis. This non-infectious atherosclerosis group had $16 \pm 13 \mathrm{IgG}^{+}$cells/hpf, $44 \pm 23 \mathrm{IgG}^{+}$cells/ hpf, and an IgG4/IgG ratio of $35 \pm 15 \%$, with none of the cases meeting staining criteria for IgG4-related disease. By ANOVA, none of the measurements were statistically different than those of the acute distal infectious aortitis group, but both the number of $\mathrm{IgG}^{+}$cells/hpf and the number of $\mathrm{IgG}^{+}$cells/hpf in the non-infectious atherosclerosis group were significantly lower than those of the chronic distal infectious aortitis group $(P=0.0008$ and $P=0.002$, respectively). 

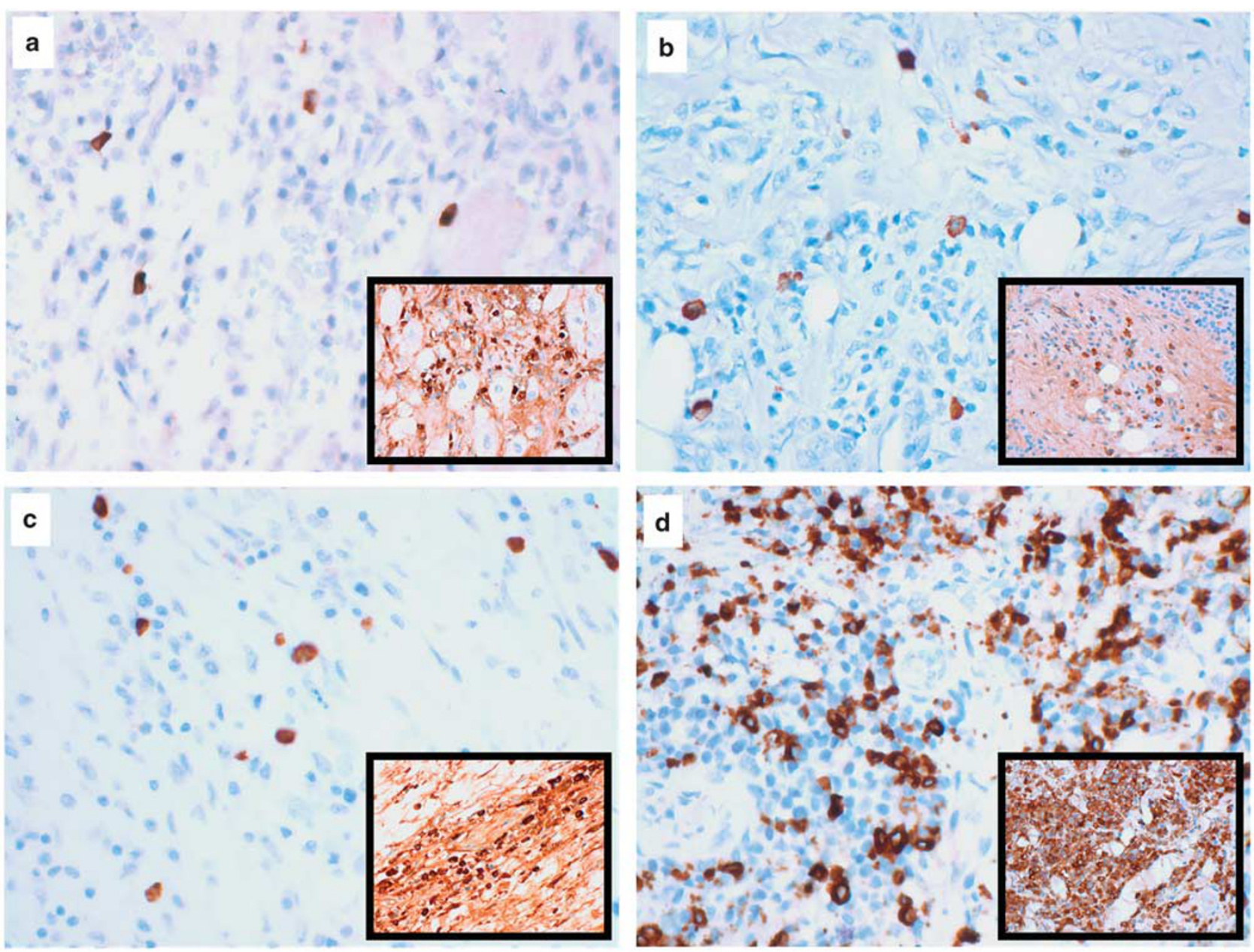

Figure 1 Immunohistochemical staining of the four infectious aortitis groups. Depicted are representative IgG4 immunohistochemical stains of the adventitia with corresponding IgG stains as insets of an acute ascending infectious aortitis (a), a chronic ascending infectious aortitis (b), an acute distal infectious aortitis (c), and a chronic distal infectious aortitis (d).

\section{Discussion}

This study demonstrates that in the setting of infectious aortitis, enhanced adventitial IgG4 ${ }^{+}$ plasma cell infiltration is associated with a prolonged symptom course of $>3$ weeks, a site of infection in the descending thoracic and abdominal aorta rather than the ascending aorta, and the presence of pre-existing moderate to severe aortic atherosclerosis. In this setting, the degree of $\operatorname{IgG} 4^{+}$ plasma cell infiltration can be intense and overlap with that of the aortitis of IgG4-related disease. The chronic time course needed for the enhanced IgG4 ${ }^{+}$ plasma cell response in infectious aortitis is not surprising. Previously lymphoplasmacytic infiltrates were identified in infectious aortitis cases showing other pathologic features of chronicity. ${ }^{13}$ In our series, there was a significant increase in $\mathrm{IgG}^{+}$plasma cells with chronicity in the distal infectious aortitis cases, and there was a non-significant trend towards increased $\mathrm{IgG}^{+}$plasma cells with chronicity in the ascending infectious aortitis cases.
The association of the enhanced IgG4 ${ }^{+}$plasma cell response in the distal aorta compared with the ascending aorta in this cohort is most likely related to the enhanced degree of pre-existing atherosclerosis in the distal aorta. There is growing evidence that atherosclerosis does enhance IgG4 inflammatory responses. Recently in a series of patients with periaortitis diagnosed by imaging, those periaortitis patients with elevated serum IgG4 levels had more aortic calcium than those periaortitis patients with normal serum IgG4 levels. ${ }^{14}$ In addition, in patients without radiologic evidence of periaortitis, increased aortic wall area by computed tomography, presumably due to atherosclerosis, has been shown to be associated with higher, albeit normal, levels of circulating IgG4. ${ }^{15}$ Furthermore, patients with coronary artery disease have been shown to have higher serum IgG4 levels than patients without coronary artery disease. ${ }^{16}$ Finally, pathologic studies both in this report and previous reports have shown atherosclerotic aortas to contain measurable but 
relatively low levels of IgG4 ${ }^{+}$plasma cells. ${ }^{3,4}$ Thus, it appears likely that atherosclerosis can promote IgG4 responses.
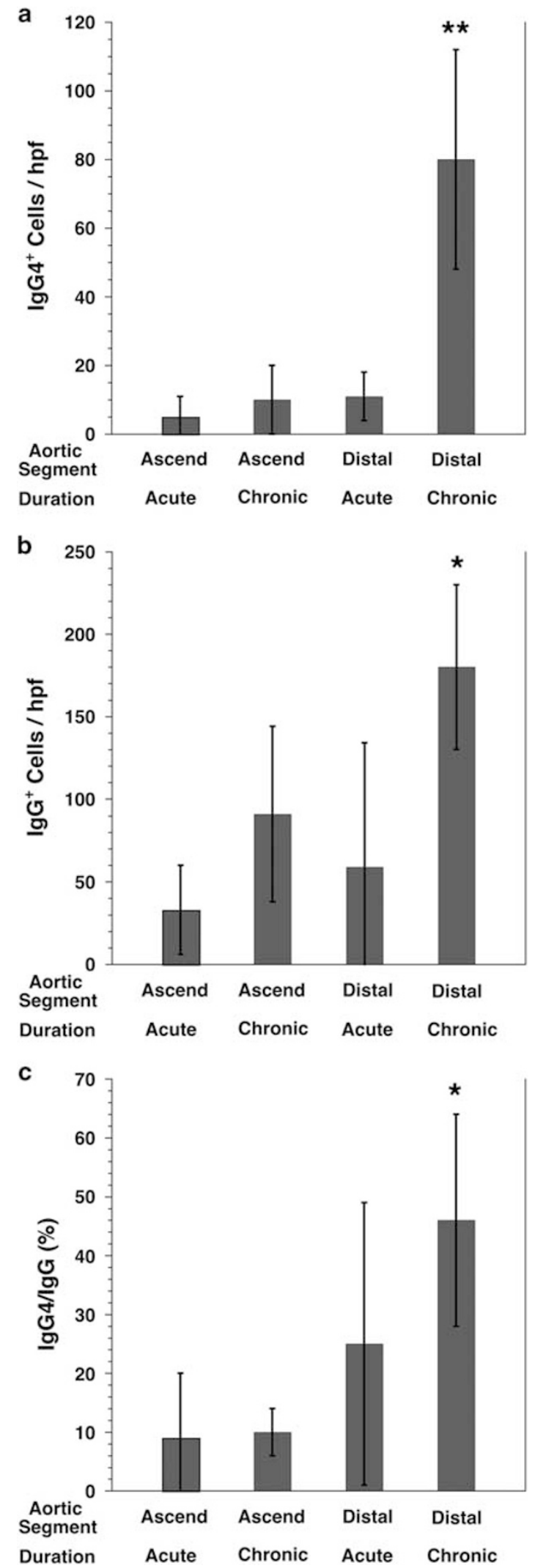

There is a substantial difference in the prevalence of IgG4-related aortitis/periaortitis when comparing the thoracic aorta with the abdominal aorta. Significant thoracic aortic disease in general affects $\sim 0.2 \%$ of the population. ${ }^{17}$ Of those thoracic aortic disease patients who undergo surgical resection, only $0.5 \%$ pathologically demonstrate IgG4-related aortitis/periaortitis, ${ }^{3}$ suggesting IgG4-related thoracic aortitis/periaortitis afflicts $~ 1$ in 100000 people. In contrast, moderate to large abdominal aortic aneurysms $(>39 \mathrm{~mm}$ ) are known to be present in about $1 \%$ of the population, ${ }^{18}$ and it has been suggested that $1-6 \%$ of these aneurysms are due to IgG4-related disease. ${ }^{6}$ This would indicate that IgG4-related aortitis/periaortitis of the abdominal aorta afflicts on the order of 1-6 of every 10000 people, making IgG4-related aortitis/periaortitis 10-60 times more common in the abdominal aorta than in the thoracic aorta. The reason for this difference may be the enhanced degree of atherosclerosis in the abdominal aorta. Many of these patients with IgG4-rich inflammatory infiltrates around the abdominal aorta do not demonstrate elevated serum IgG4 levels or show overt features of a systemic vasculitis.

Even though atherosclerosis likely promotes IgG4 responses, most patients with aortic atherosclerosis do not develop an intense IgG4 response to the aortic plaque., ${ }^{3,4}$ Thus, other factors beyond just atherosclerosis appear to be involved for this to occur. Our data indicate that one such factor is the chronic infection of the plaque by bacteria. There may be other such factors that are yet to be identified. However, this response to infection has important implications for the etiology of $\mathrm{IgG}^{+}$plasma cell infiltrates in inflammatory atherosclerotic aortic aneurysms. Once the infection has been cleared and the suppurative inflammation dissipates, if the enhanced IgG4 ${ }^{+}$plasma cell infiltrate persists, then the pathology would be suggestive of IgG4-related aortitis/periaortitis. IgG4-related periaortitis has been reported to be present in up to $40 \%$ of inflammatory atherosclerotic aortic aneurysms. ${ }^{6}$ Thus, there exists the distinct possibility, that some cases of abdominal IgG4-related aortitis/periaortitis result from an occult and/or potentially resolved bacterial infection of a pre-existing atherosclerotic aneurysm.

For the chronic infectious aortitis cases reported here, the suppurative pattern of inflammation extensively involved the atherosclerotic plaque, but it also

\footnotetext{
Figure 2 Quantification of IgG4 and IgG staining. (a) Shown are the absolute numbers of IgG4 ${ }^{+}$cells per hpf for the four aortitis groups. ${ }^{*} P \leq 0.0007$ versus the other three groups. (b) Shown are the absolute numbers of $\mathrm{IgG}^{+}$cells for the four aortitis groups. ${ }^{*} P \leq 0.03$ versus the acute ascending and acute distal groups. (c) Shown are the IgG4/IgG cell count ratios for the four aortitis groups. ${ }^{*} P \leq 0.03$ versus the acute ascending and chronic ascending groups. $P$-values were derived from ANOVA with Bonferroni post-test. All other comparisons demonstrated no statistically significant difference. All values represent mean \pm s.d.
} 

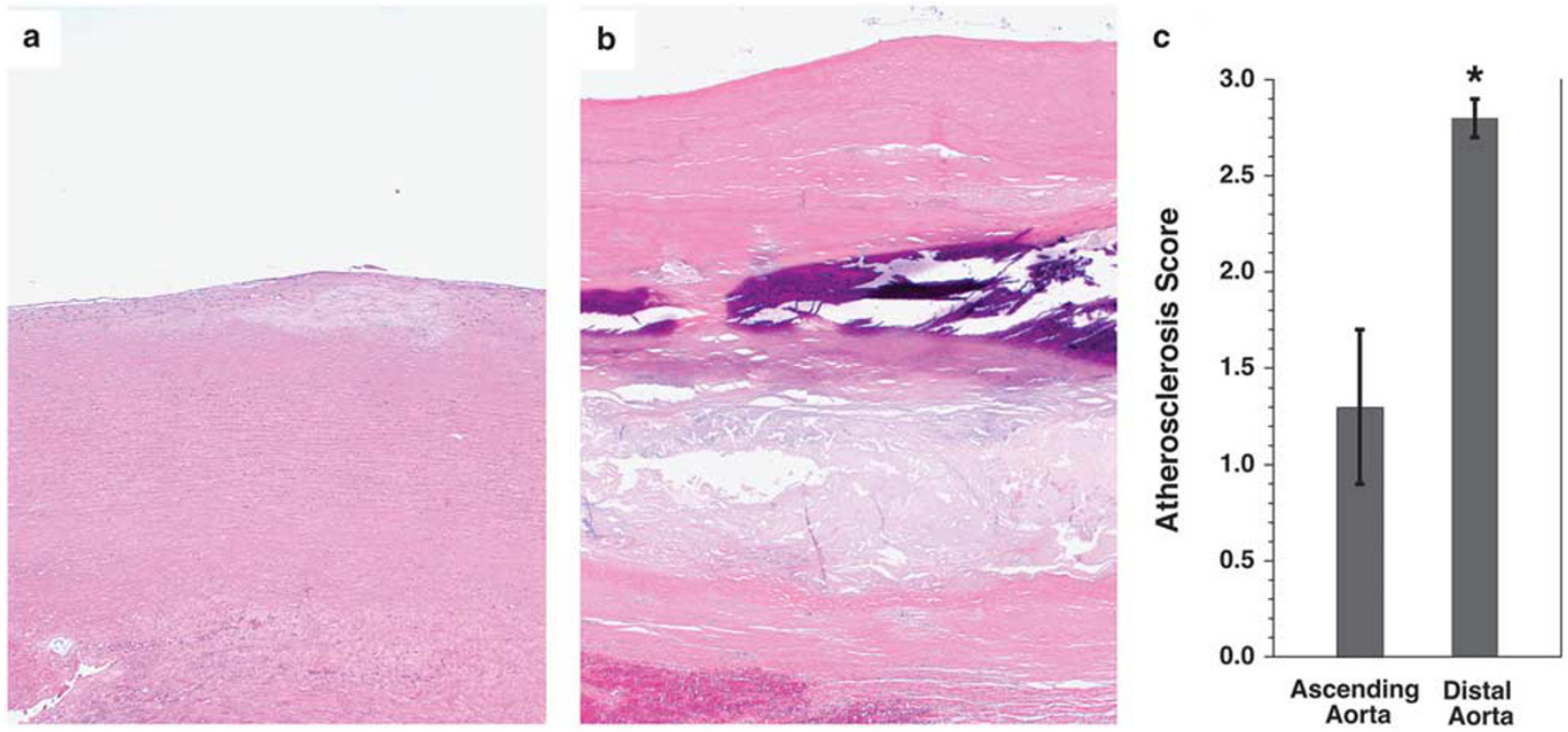

Figure 3 Atherosclerosis in the ascending and distal aorta. (a) Depicted is a histologic image showing mild atherosclerosis in the ascending aorta. (b) Depicted is a histologic image showing severe atherosclerosis in the distal aorta. (c) Comparison of atherosclerosis severity in the ascending aorta $(n=8)$ with atherosclerosis severity in the distal aorta $(n=9)$. Data represent mean \pm s.e. ${ }^{*} P=0.008$ MannWhitney $U$-test.

involved the adventitia in all five cases, making these cases readily distinguishable from both IgG4related aortitis/periaortitis and inflammatory atherosclerotic aneurysms. In all of these cases, the lymphoplasmacytic component was predominantly in the adventitia. In one case, the lymphoplasmacytic component was restricted to the adventitia analogous to IgG4-related periaortitis, but in the other four cases, the lymphoplasmacytic component also focally involved the media analogous to the adventitial-predominant form of IgG4-related aortitis. However, in true IgG4-related aortitis/periaortitis the extent of the dense IgG4 $4^{+}$plasma cell infiltration is usually more widespread than in chronic infectious aortitis, where the areas of dense $\mathrm{IgG} 4^{+}$plasma cells are relatively focal. Also, some but not all cases of IgG4-related aortitis/periaortitis will display a storiform pattern of fibrosis with a pinwheel pattern. Such a pattern of fibrosis was not present in the chronic infectious aortitis cases. If the suppurative inflammation was no longer present, the five chronic infectious aortitis cases would meet histologic criteria for inflammatory atherosclerotic aneurysms, although only four of the five cases were actually grossly aneurysmal. For these five cases the aortic wall thickness was $7.6 \pm 1.5 \mathrm{~mm}$ (mean \pm s.d.), with all cases exceeding $4 \mathrm{~mm}$, the minimal requirement for inflammatory atherosclerotic aneurysms. ${ }^{1}$

It is recommended that chronic active infectious aortitis cases, such as the ones presented here, be reported as aortitis with a suppurative pattern of inflammation. ${ }^{1}$ It is also recommended that the results of stains for microorganisms be included, and that it be made clear that the pathology is a result of infection. Quantification of the IgG4 ${ }^{+}$plasma cell response is probably not necessary in this setting. However, if $\mathrm{IgG4}^{+}$plasma cell quantification is performed on such a case and is elevated, it should be made clear that the elevated $\operatorname{IgG} 4^{+}$plasma cell infiltration is most likely due to the chronic infection and not due to IgG4-related disease. Conversely, when an elevated $\mathrm{IgG}^{+}$plasma cell response is identified in an aortic specimen with severe atherosclerosis, it is recommended to evaluate multiple tissue blocks (up to 12) for foci of suppurative inflammation, which if present should be assessed with stains for microorganisms to determine if there is a chronic/resolving infection. ${ }^{1,9}$

\section{Disclosure/conflict of interest}

The authors declare no conflict of interest.

\section{References}

1 Stone JR, Bruneval P, Angelini A et al. Consensus statement on surgical pathology of the aorta from the Society for Cardiovascular Pathology and the Association for European Cardiovascular Pathology: I. Inflammatory diseases. Cardiovasc Pathol pii: S1054-8807(15)00056-3.

2 Kashashima S, Zen Y, Kawashima A et al. A clinicopathologic study of immunoglobulin G4-related sclerosing disease of the thoracic aorta. J Vasc Surg 2010;52: 1587-1595.

3 Stone JH, Khosroshahi A, Deshpande V et al. IgG4-related systemic disease accounts for a significant proportion of thoracic lymphoplasmacytic aortitis cases. Arthrit Care Res 2010;62:316-322.

4 Kasashima S, Zen Y, Kawashima A et al. A new clinicopathological entity of IgG4-related inflammatory 
abdominal aortic aneurysm. J Vasc Surg 2009;49: 1264-1271.

5 Stone JH, Khosroshahi A, Hilgenberg A et al. IgG4related systemic disease and lymphoplasmacytic aortitis. Arthrit Rheum 2009;60:3139-3145.

6 Stone JR. Aortitis, periaortitis, and retroperitoneal fibrosis as manifestations of IgG4-related systemic disease. Curr Opin Rheumatol 2011;23:88-94.

7 Siddiquee Z, Zane NA, Smith RN et al. Dense IgG4 plasma cell infiltrates associated with chronic infectious aortitis: implications for the diagnosis of IgG4-related disease. Cardiovasc Pathol 2012;21: $470-475$.

8 Khosroshahi A, Bloch DB, Deshpande V et al. Rituximab therapy leads to rapid decline of serum IgG4 levels and prompt clinical improvement in IgG4-related systemic disease. Arthrit Rheum 2010;62:1755-1762.

9 Stone JR, Basso C, Baandrup UT et al. Recommendations for processing cardiovascular surgical pathology specimens: a consensus statement from the Standards and Definitions Committee of the Society for Cardiovascular Pathology and the Association for European Cardiovascular Pathology. Cardiovasc Pathol 2012;21: 2-16.

10 Baggett MV, Turbett SE, Schwartzenberg SS et al. A 59year-old man with fever, confusion, thrombocytopenia, rash, and renal failure. N Engl J Med 2014;370:651-660.

11 Deshpande V, Sainani NI, Chung RT et al. IgG4associated cholangitis: a comparative histological and immunophenotypic study with primary sclerosing cholangitis on liver biopsy material. Mod Pathol 2009;22:1287-1295.

12 Deshpande V, Zen Y, Chan JK et al. Consensus statement on the pathology of IgG4-related disease. Mod Pathol 2012;25:1181-1192.

13 Miller DV, Oderich GS, Aubury M-C et al. Surgical pathology of infected aneurysms of the descending thoracic and abdominal aorta: clinicopathologic correlations in 29 cases (1976 to 1999). Hum Pathol 2004;35: 1112-1120.

14 Castelein T, Coudyzer W, Blockmans D. IgG4-related periaortitis vs idiopathic periaortitis: is there a role for atherosclerotic plaque in the pathogenesis of IgG4related periaortitis? Rheumatology 2015;54:1250-1256.

15 Sakamoto A, Ishizaka N, Imai Y et al. Association between serum IgG4 concentrations and the morphology of the aorta in patients who undergo cardiac computed tomography. J Cardiol 2015;65:150-156.

16 Sakamoto A, Ishizaka N, Saito K et al. Serum levels of IgG4 and soluble interleukin-2 receptor in patients with coronary artery disease. Clin Chim Acta 2012;413: 577-581.

17 Clouse WD, Hallett JW, Schaff HV et al. Acute aortic dissection: Population-based incidence compared with degenerative aortic aneurysm rupture. Mayo Clin Proc 2004;79:176-180.

18 Singh K, Bonaa KH, Jacobsen BK et al. Prevalence of and risk factors for abdominal aortic aneurysms in a population-based study: The Tromso Study. Am J Epidemiol 2001;154:236-244. 